IPM/P-2008/067

\title{
More on WZ action of non-BPS branes
}

\author{
Mohammad R. Garousi and Ehsan Hatefi \\ Department of Physics, Ferdowsi University of Mashhad, \\ P.O. Box 1436, Mashhad, Iran \\ School of Physics, Institute for research in fundamental sciences (IPM), \\ P.O.Box 19395-5531, Tehran, Iran \\ E-mails : garousi@mail.ipm.ir, hatefi@ipm.ir
}

\begin{abstract}
We calculate the disk level S-matrix element of one Ramond-Ramond, two gauge field and one tachyon vertex operators in the world volume of non-BPS branes. We then find the momentum expansion of this amplitude and show that the infinite tachyon/massless poles and the contact terms of this amplitude can be reproduced by the tachyon DBI and the WZ actions, and by their higher derivative corrections.
\end{abstract}




\section{Introduction}

Spectrum of non-BPS branes has tachyon, massless and infinite tower of massive states. Even though the mass scale of tachyon and the massive states are the same, there are various arguments that indicate there must be an effective theory for non-BPS branes that includes only the tachyon and the massless states [1]. The effective theory should have two parts, i.e.,

$$
S_{n o n-B P S}=S_{D B I}+S_{W Z}
$$

where $S_{D B I} / S_{W Z}$ should be an extension of DBI/WZ action of BPS branes in which the tachyon mode of non-BPS brane are included appropriately.

One method for finding these effective actions is the BSFT. In this formalism the kinetic term of tachyon appears in the DBI part as [2, 3]

$$
S_{D B I} \sim \int d^{p+1} \sigma e^{-2 \pi T^{2}}\left(F\left(2 \pi \alpha^{\prime} D^{a} T D_{a} T\right)+\cdots\right), \quad F(x)=\frac{4^{x} x \Gamma(x)^{2}}{2 \Gamma(2 x)}
$$

where dots refer to the gauge field couplings. When tachyon is zero, they are given by the DBI action. The WZ term in this formalism is given by [2, 3]

$$
S_{W Z}=\mu_{p}^{\prime} \int_{\Sigma_{(p+1)}} C \wedge \operatorname{Str} e^{i 2 \pi \alpha^{\prime} \mathcal{F}}
$$

in which the curvature of superconnection is

$$
i \mathcal{F}=\left(\begin{array}{cc}
i F-\beta^{\prime 2} T^{2} & \beta^{\prime} D T \\
\beta^{\prime} D T & i F-\beta^{\prime 2} T^{2}
\end{array}\right)
$$

and $\beta^{\prime}$ is a normalization constant with dimension $1 / \sqrt{\alpha^{\prime}}$.

Another method for studying the effective action of non-BPS branes is the S-matrix method. In this formalism the kinetic term of tachyon appear in the DBI action as [4, 6]

$$
\begin{aligned}
& S_{D B I} \sim \int d^{p+1} \sigma \operatorname{STr}\left(V\left(T^{i} T^{i}\right) \sqrt{\left.1+\frac{1}{2}\left[T^{i}, T^{j}\right]\left[T^{j}, T^{i}\right]\right)}\right. \\
&\left.\times \sqrt{-\operatorname{det}\left(\eta_{a b}+2 \pi \alpha^{\prime} F_{a b}+2 \pi \alpha^{\prime} D_{a} T^{i}\left(Q^{-1}\right)^{i j} D_{b} T^{j}\right)}\right),
\end{aligned}
$$

where $V\left(T^{i} T^{i}\right)=e^{-\pi T^{i} T^{i} / 2}$, and

$$
Q^{i j}=I \delta^{i j}-i\left[T^{i}, T^{j}\right]
$$


The superscripts $i, j=1,2$, i.e., $T^{1}=T \sigma_{1}, T^{2}=T \sigma_{2}$ and there is no sum over $i, j . \sigma_{1}$ and $\sigma_{2}$ are the Pauli matrices. After expanding the square roots one should choose two of the tachyons to be $T^{2}$ and the others to be $T^{1}$. The trace in above equation must be completely symmetric between all matrices of the form $F_{a b}, D_{a} T^{i},\left[T^{i}, T^{j}\right]$ and individual $T^{i}$ of the potential $V\left(T^{i} T^{i}\right)$. The above action is consistent with the momentum expansion of the S-matrix element of four tachyons, the S-matrix element of one RR and three tachyons [4, 6] and with the momentum expansion of the S-matrix element of four tachyons and one gauge field [7]. Around the stable point of the tachyon potential, the above action reduces to the usual tachyon DBI action [8, 9, 10, 11] with potential $T^{4} V\left(T^{2}\right)$. The WZ part in this formalism, on the other hand, is given by the same WZ action as in the BSFT in which the normalization of tachyon is $\beta^{\prime}=\frac{1}{\pi} \sqrt{\frac{6 \ln (2)}{\alpha^{\prime}}}$ for non-BPS branes [4] and $\beta=\frac{1}{\pi} \sqrt{\frac{2 \ln (2)}{\alpha^{\prime}}}$

for brane-anti-brane [5]. In fact the structure of superconnection in the WZ part has been found first by the S-matrix method in [12].

In this paper, we would like to examine the above actions with the S-matrix element of one RR, two gauge fields and one tachyon. So in the next section we calculate this S-matrix element in string theory. In section 3, we find the momentum expansion of the amplitude and compare it with the various Feynman amplitudes resulting from the couplings in the above actions and their higher derivative extensions.

\section{The four-point amplitude}

To calculate a S-matrix element, one needs to choose the picture of the vertex operators appropriately. The sum of the superghost charge must be -2 for disk level amplitude. On the other hand, the vertex operators of a non-BPS D-brane carry internal CP matrix [13]. Using the fact that the picture changing operator also carries internal CP matrix $\sigma_{3}$ [14], one realizes that a vertex operator carries different internal $\mathrm{CP}$ matrices depending on its superghost charges. It has been speculated in [6] that the S-matrix element of $n$ external states is independent of the choice of the picture of the external states only when one includes the internal CP matrix in the vertex operators.

When tachyon is set to zero, the effective field theory of non-BPS branes/brane-antibrane must reduce to the effective field theory of BPS branes in which there is no internal CP matrix. This indicates that the massless fields in the effective field theory of non-BPS branes/brane-antibrane must carry identity internal CP matrix. For example, the RR field in the effective field theory of brane-antibrane must carry identity matrix because when 
tachyon is set to zero the WZ action of brane-antibrane reduces to the WZ action of two stable BPS branes. This internal CP matrix is contributed to the RR vertex operator in (0)picture. In other pictures, the internal CP matrix is different depending on its superghost picture. It has been shown in [13] that the RR vertex operator of a non-BPS brane in (0)-picture must carry the internal $\mathrm{CP}$ matrix $\sigma_{1}$. This vertex operator in $(-1)$-picture then must carry the internal matrix $\sigma_{3} \sigma_{1}$.

Hence, the S-matrix element of one RR field, two gauge fields and one tachyon in the world volume of non-BPS branes is given by the following correlation function:

$$
\mathcal{A}^{A A T C} \sim \sum_{\text {non-cyclic }} \int d x_{1} d x_{2} d x_{3} d z d \bar{z}\left\langle V_{A}^{(0)}\left(x_{1}\right) V_{A}^{(0)}\left(x_{2}\right) V_{T}^{(-2)}\left(x_{3}\right) V_{R R}^{(0)}(z, \bar{z})\right\rangle
$$

The internal CP factor is $\operatorname{Tr}\left(I I \sigma_{1} \sigma_{1}\right)=2$ for 123 ordering and is $\operatorname{Tr}\left(I \sigma_{1} I \sigma_{1}\right)=2$ for 132 ordering. The internal CP matrix of tachyon in (-2)-picture is the same as the CP matrix of this operator in (0)-picture which is $\sigma_{1}$. It is easier to calculate the S-matrix element in the following picture:

$$
\mathcal{A}^{A A T C} \sim \sum_{\text {non-cyclic }} \int d x_{1} d x_{2} d x_{3} d z d \bar{z}\left\langle V_{A}^{(0)}\left(x_{1}\right) V_{A}^{(0)}\left(x_{2}\right) V_{T}^{(-1)}\left(x_{3}\right) V_{R R}^{(-1)}(z, \bar{z})\right\rangle
$$

The CP factor in this case is $\operatorname{Tr}\left(I I \sigma_{2} \sigma_{3} \sigma_{1}\right)=2 i$ for 123 ordering and is $\operatorname{Tr}\left(I \sigma_{2} I \sigma_{3} \sigma_{1}\right)=2 i$ for 132 ordering. According to the speculation in [6], the difference between the above two S-matrix elements is a factor of $i$ when the internal CP factors are included. So to calculate the S-matrix element (5) in which the vertexes carry the same internal CP matrices as the effective field theory, we first calculate the amplitude (6) and then multiply the result by $i$.

The vertex operators in (6) are given a: 1

$$
\begin{aligned}
V_{T}^{(-1)}(y) & =e^{-\phi(y)} e^{2 i k \cdot X(y)} \lambda \otimes \sigma_{2} \\
V_{A}^{(0)}(x) & =\xi_{i}\left(\partial X^{i}(x)+2 i k \cdot \psi \psi^{i}(x)\right) e^{2 i k \cdot X(x)} \lambda \otimes I \\
V_{R R}^{(-1)}(z, \bar{z}) & =\left(P_{-} H_{(n)} M_{p}\right)^{\alpha \beta} e^{-\phi(z) / 2} S_{\alpha}(z) e^{i p \cdot X(z)} e^{-\phi(\bar{z}) / 2} S_{\beta}(\bar{z}) e^{i p \cdot D \cdot X(\bar{z})} \otimes \sigma_{3} \sigma_{1}
\end{aligned}
$$

where $k$ is the momentum of open string that for tachyon satisfies the on-shell condition $k^{2}=1 / 4$, and $\lambda$ is the external CP matrix in the $U(N)$ group. We refer the interested reader to [15] for our conventions on the $R R$ vertex operator.

To calculate the correlators in (6), one must use the following standard propagators:

$$
\left\langle X^{\mu}(z) X^{\nu}(w)\right\rangle=-\eta^{\mu \nu} \log (z-w)
$$

\footnotetext{
${ }^{1}$ In string theory side, our conventions set $\alpha^{\prime}=2$.
} 


$$
\begin{aligned}
\left\langle\psi^{\mu}(z) \psi^{\nu}(w)\right\rangle & =-\eta^{\mu \nu}(z-w)^{-1} \\
\langle\phi(z) \phi(w)\rangle & =-\log (z-w)
\end{aligned}
$$

Introducing $x_{4} \equiv z=x+i y$ and $x_{5} \equiv \bar{z}=x-i y$, the amplitude factorizes to the following correlators for 123 ordering:

$$
\begin{aligned}
\mathcal{A}^{A A T C} \sim & \int d x_{1} d x_{2} d x_{3} d x_{4} d x_{5}\left(P_{-} H_{(n)} M_{p}\right)^{\alpha \beta} \xi_{1 i} \xi_{2 j} x_{45}^{-1 / 4} x_{43}^{-1 / 2} x_{53}^{-1 / 2}\left(I_{1}+I_{2}+I_{3}+I_{4}\right) \\
& \times \operatorname{Tr}\left(\lambda_{1} \lambda_{2} \lambda_{3}\right) \operatorname{Tr}\left(I I \sigma_{2} \sigma_{3} \sigma_{1}\right)
\end{aligned}
$$

where $x_{i j}=x_{i}-x_{j}$ and

$$
\begin{aligned}
I_{1}= & <: \partial X^{i}\left(x_{1}\right) e^{2 i k_{1} \cdot X\left(x_{1}\right)}: \partial X^{j}\left(x_{2}\right) e^{2 i k_{2} \cdot X\left(x_{2}\right)}: e^{2 i k_{3} \cdot X\left(x_{3}\right)}: e^{i p . X\left(x_{4}\right)}: e^{i p . D . X\left(x_{5}\right)}:> \\
& \times<S_{\alpha}\left(x_{4}\right): S_{\beta}\left(x_{5}\right):> \\
I_{2}= & <: \partial X^{i}\left(x_{1}\right) e^{2 i k_{1} \cdot X\left(x_{1}\right)}: e^{2 i k_{2} \cdot X\left(x_{2}\right)}: e^{2 i k_{3} \cdot X\left(x_{3}\right)}: e^{i p . X\left(x_{4}\right)}: e^{i p . D . X\left(x_{5}\right)}:> \\
& \times<: S_{\alpha}\left(x_{4}\right): S_{\beta}\left(x_{5}\right): 2 i k_{2} \cdot \psi \psi^{j}\left(x_{2}\right):> \\
I_{3}= & <: e^{2 i k_{1} \cdot X\left(x_{1}\right)}: \partial X^{j}\left(x_{2}\right) e^{2 i k_{2} \cdot X\left(x_{2}\right)}: e^{2 i k_{3} \cdot X\left(x_{3}\right)}: e^{i p . X\left(x_{4}\right)}: e^{i p . D . X\left(x_{5}\right)}:> \\
& \times<: S_{\alpha}\left(x_{4}\right): S_{\beta}\left(x_{5}\right): 2 i k_{1} \cdot \psi \psi^{i}\left(x_{1}\right):> \\
I_{4}= & <: e^{2 i k_{1} \cdot X\left(x_{1}\right)}: e^{2 i k_{2} \cdot X\left(x_{2}\right)}: e^{2 i k_{3} \cdot X\left(x_{3}\right)}: e^{i p . X\left(x_{4}\right)}: e^{i p . D . X\left(x_{5}\right)}:> \\
& \times<: S_{\alpha}\left(x_{4}\right): S_{\beta}\left(x_{5}\right): 2 i k_{1} \cdot \psi \psi^{i}\left(x_{1}\right): 2 i k_{2} \cdot \psi \psi^{j}\left(x_{2}\right):>
\end{aligned}
$$

One can perform easily the correlators of $X$ using the corresponding propagator in (7). To find the correlator of $\psi$, we use the following Wick-like rule [16] for the correlation function involving an arbitrary number of $\psi$ 's and two $S$ 's:

$$
\begin{aligned}
\left\langle\psi^{\mu_{1}}\left(y_{1}\right) \ldots \psi^{\mu_{n}}\left(y_{n}\right) S_{\alpha}(z) S_{\beta}(\bar{z})\right\rangle= & \frac{1}{2^{n / 2}} \frac{(z-\bar{z})^{n / 2-5 / 4}}{\left|y_{1}-z\right| \ldots\left|y_{n}-z\right|}\left[\left(\Gamma^{\mu_{n} \ldots \mu_{1}} C^{-1}\right)_{\alpha \beta}\right. \\
& +\left\langle\left\langle\psi^{\mu_{1}}\left(y_{1}\right) \psi^{\mu_{2}}\left(y_{2}\right)\right\rangle\right\rangle\left(\Gamma^{\mu_{n} \ldots \mu_{3}} C^{-1}\right)_{\alpha \beta} \pm \text { perms } \\
& +\left\langle\left\langle\psi^{\mu_{1}}\left(y_{1}\right) \psi^{\mu_{2}}\left(y_{2}\right)\right\rangle\right\rangle\left\langle\left\langle\psi^{\mu_{3}}\left(y_{3}\right) \psi^{\mu_{4}}\left(y_{4}\right)\right\rangle\right\rangle\left(\Gamma^{\mu_{n} \ldots \mu_{5}} C^{-1}\right)_{\alpha \beta} \\
& \pm \text { perms }+\cdots]
\end{aligned}
$$

where dots mean sum over all possible contractions. In above equation, $\Gamma^{\mu_{n} \ldots \mu_{1}}$ is the totally antisymmetric combination of the gamma matrices and the Wick-like contraction is given by

$$
\begin{aligned}
\left\langle\left\langle\psi^{\mu}\left(y_{1}\right) \psi^{\nu}\left(y_{2}\right)\right\rangle\right\rangle & =\eta^{\mu \nu} \frac{\left(y_{1}-z\right)\left(y_{2}-\bar{z}\right)+\left(y_{2}-z\right)\left(y_{1}-\bar{z}\right)}{\left(y_{1}-y_{2}\right)(z-\bar{z})} \\
& =2 \eta^{\mu \nu} \frac{\operatorname{Re}\left[\left(y_{1}-z\right)\left(y_{2}-\bar{z}\right)\right]}{\left(y_{1}-y_{2}\right)(z-\bar{z})}
\end{aligned}
$$


where in the second line the fact that $y_{1}, y_{2}$ are real has been used. One can use the above formula to find the correlation function of two $S$ 's and an arbitrary number of currents. The only subtlety in using the formula (9) for currents is that one must not consider the Wick-like contraction for the two $\psi$ 's in one current. Using this, one can easily find the following standard results when there is no current and when there is one current:

$$
\begin{aligned}
<: S_{\alpha}\left(x_{4}\right): S_{\beta}\left(x_{5}\right):> & =x_{45}^{-5 / 4} C_{\alpha \beta}^{-1} \\
<: S_{\alpha}\left(x_{4}\right): S_{\beta}\left(x_{5}\right): \psi^{m} \psi^{i}\left(x_{1}\right):> & =-\frac{1}{2} x_{45}^{-1 / 4} x_{14}^{-1} x_{15}^{-1}\left(\Gamma^{m i} C^{-1}\right)_{\alpha \beta}
\end{aligned}
$$

In the second relation, we have not consider the Wick-like contraction for the two $\psi$ 's since both of them belong to one current. When there are two currents, the formula (9) gives the following result:

$$
\begin{aligned}
I_{4}^{\prime}= & <: S_{\alpha}\left(x_{4}\right): S_{\beta}\left(x_{5}\right): \psi^{m} \psi^{i}\left(x_{1}\right): \psi^{l} \psi^{j}\left(x_{2}\right):> \\
= & \frac{1}{4} x_{45}^{3 / 4}\left(x_{14} x_{15} x_{24} x_{25}\right)^{-1}\left\{\left(\Gamma^{j l i m} C^{-1}\right)_{\alpha \beta}+2 \frac{\operatorname{Re}\left[x_{14} x_{25}\right]}{x_{12} x_{45}}\left[\eta^{m l}\left(\Gamma^{j i} C^{-1}\right)_{\alpha \beta}-\eta^{m j}\left(\Gamma^{l i} C^{-1}\right)_{\alpha \beta}\right.\right. \\
& \left.\left.-\eta^{i l}\left(\Gamma^{j m} C^{-1}\right)_{\alpha \beta}+\eta^{i j}\left(\Gamma^{l m} C^{-1}\right)_{\alpha \beta}\right]+4\left(\frac{\operatorname{Re}\left[x_{14} x_{25}\right]}{x_{12} x_{45}}\right)^{2}\left(-\eta^{m l} \eta^{i j}+\eta^{m j} \eta^{i l}\right) C_{\alpha \beta}^{-1}\right\}
\end{aligned}
$$

Replacing the above spin correlators into (8) and performing the correlators over $X$, one finds

$$
\begin{aligned}
\mathcal{A}^{A A T C} \sim & \int d x_{1} d x_{2} d x_{3} d x_{4} d x_{5}\left(P_{-} H_{(n)} M_{p}\right)^{\alpha \beta} I \xi_{1 i} \xi_{2 j} x_{45}^{-1 / 4} x_{43}^{-1 / 2} x_{53}^{-1 / 2} \\
& \times\left(x_{45}^{-5 / 4} C_{\alpha \beta}^{-1}\left(-\eta^{i j} x_{12}^{-2}+a_{1}^{i} a_{2}^{j}\right)+a_{1}^{i}\left(a_{3}^{j}\right)_{\alpha \beta}+a_{2}^{j}\left(a_{4}^{i}\right)_{\alpha \beta}-4 k_{1 m} k_{2 l} I_{4}^{\prime}\right)
\end{aligned}
$$

where $I_{4}^{\prime}$ is given in (12), and

$$
\begin{aligned}
I & =\left|x_{12}\right|^{4 k_{1} \cdot k_{2}}\left|x_{13}\right|^{4 k_{1} \cdot k_{3}}\left|x_{14} x_{15}\right|^{2 k_{1} \cdot p}\left|x_{23}\right|^{4 k_{2} \cdot k_{3}}\left|x_{24} x_{25}\right|^{2 k_{2} \cdot p}\left|x_{34} x_{35}\right|^{2 k_{3} \cdot p}\left|x_{45}\right|^{p . D \cdot p} \\
a_{1}^{i} & =-i k_{2}^{i}\left(\frac{x_{42}}{x_{41} x_{12}}+\frac{x_{52}}{x_{51} x_{12}}\right)-i k_{3}^{i}\left(\frac{x_{43}}{x_{41} x_{13}}+\frac{x_{53}}{x_{51} x_{13}}\right) \\
a_{2}^{j} & =-i k_{1}^{j}\left(\frac{x_{14}}{x_{42} x_{12}}+\frac{x_{15}}{x_{52} x_{12}}\right)-i k_{3}^{j}\left(\frac{x_{43}}{x_{42} x_{23}}+\frac{x_{53}}{x_{52} x_{23}}\right) \\
\left(a_{3}^{j}\right)_{\alpha \beta} & =-i k_{2 l} x_{45}^{-1 / 4}\left(\Gamma^{l j} C^{-1}\right)_{\alpha \beta}\left(x_{24} x_{25}\right)^{-1} \\
\left(a_{4}^{i}\right)_{\alpha \beta} & =-i k_{1 m} x_{45}^{-1 / 4}\left(\Gamma^{m i} C^{-1}\right)_{\alpha \beta}\left(x_{14} x_{15}\right)^{-1}
\end{aligned}
$$

One can show that the integrand is invariant under $\operatorname{SL}(2, \mathrm{R})$ transformation. Gauge fixing this symmetry by fixing the position of the open string vertex operators as

$$
x_{1}=0, \quad x_{2}=1, \quad x_{3} \rightarrow \infty
$$


One finds the following integral:

$$
\int d^{2} z|1-z|^{a}|z|^{b}(z-\bar{z})^{c}(z+\bar{z})^{d}
$$

where $d=0,1,2$ and $a, b, c$ are given in terms of the Mandelstam variables:

$$
s=-\left(k_{1}+k_{3}\right)^{2}, \quad t=-\left(k_{1}+k_{2}\right)^{2}, \quad u=-\left(k_{2}+k_{3}\right)^{2}
$$

The region of integration is the upper half complex plane. For $d=0,1$ the result is given in [17], i.e.,

$$
\int d^{2} z|1-z|^{a}|z|^{b}(z-\bar{z})^{c}(z+\bar{z})^{d}=(2 i)^{c} 2^{d} \pi \frac{\Gamma\left(1+d+\frac{b+c}{2}\right) \Gamma\left(1+\frac{a+c}{2}\right) \Gamma\left(-1-\frac{a+b+c}{2}\right) \Gamma\left(\frac{1+c}{2}\right)}{\Gamma\left(-\frac{a}{2}\right) \Gamma\left(-\frac{b}{2}\right) \Gamma\left(2+c+d+\frac{a+b}{2}\right)}
$$

Extending the result in [17] to $d=2$, one finds

$$
\int d^{2} z|1-z|^{a}|z|^{b}(z-\bar{z})^{c}(z+\bar{z})^{d}=(2 i)^{c} 2^{d} \pi \frac{J_{1}+J_{2}}{\Gamma\left(-\frac{a}{2}\right) \Gamma\left(-\frac{b}{2}\right) \Gamma\left(d+2+c+\frac{a+b}{2}\right)}
$$

where

$$
\begin{aligned}
& J_{1}=\frac{1}{2} \Gamma\left(d+\frac{b+c}{2}\right) \Gamma\left(d+\frac{a+c}{2}\right) \Gamma\left(-d-\frac{a+b+c}{2}\right) \Gamma\left(\frac{1+c}{2}\right) \\
& J_{2}=\Gamma\left(d+1+\frac{b+c}{2}\right) \Gamma\left(1+\frac{a+c}{2}\right) \Gamma\left(-1-\frac{a+b+c}{2}\right) \Gamma\left(\frac{1+c}{2}\right)
\end{aligned}
$$

Using the above integrals, one can write the amplitude (13) as

$$
\mathcal{A}^{A A T C}=\mathcal{A}_{1}+\mathcal{A}_{2}+\mathcal{A}_{3}
$$

where

$$
\begin{aligned}
\mathcal{A}_{1} \sim & -2 i \operatorname{Tr}\left(\lambda_{1} \lambda_{2} \lambda_{3}\right) \xi_{1 i} \xi_{2 j} k_{1 m} k_{2 l} \operatorname{Tr}\left(P_{-} H_{(n)} M_{p} \Gamma^{j l i m}\right)(t+s+u+1 / 2) L_{3} \\
\mathcal{A}_{2} \sim & 2 \operatorname{Tr}\left(\lambda_{1} \lambda_{2} \lambda_{3}\right)\left\{\left[k_{2 l} \xi_{2 j}\left(-2 k_{2} \cdot \xi_{1} L_{1}+2 k_{3} \cdot \xi_{1} L_{2}\right) \operatorname{Tr}\left(P_{-} H_{(n)} M_{p} \Gamma^{l j}\right)\right.\right. \\
& \left.-2 k_{1} \cdot \xi_{2} k_{2 l} \xi_{1 i} \operatorname{Tr}\left(P_{-} H_{(n)} M_{p} \Gamma^{i l}\right) L_{1}\right]-[1 \leftrightarrow 2] \\
& \left.-L_{1}\left(-t \xi_{1 i} \xi_{2 j} \operatorname{Tr}\left(P_{-} H_{(n)} M_{p} \Gamma^{j i}\right)+2 k_{2 l} k_{1 m} \xi_{1} \cdot \xi_{2} \operatorname{Tr}\left(P_{-} H_{(n)} M_{p} \Gamma^{l m}\right)\right)\right\} \\
\mathcal{A}_{3} \sim \quad & -2 i \operatorname{Tr}\left(\lambda_{1} \lambda_{2} \lambda_{3}\right) \operatorname{Tr}\left(P_{-} H_{(n)} M_{p}\right) L_{3}\left[-t\left(k_{3} \cdot \xi_{1}\right)\left(k_{3} \cdot \xi_{2}\right)+\left(k_{3} \cdot \xi_{2}\right)\left(k_{2} \cdot \xi_{1}\right)\left(s+\frac{1}{4}\right)\right. \\
& \left.+\left(k_{3} \cdot \xi_{1}\right)\left(k_{1} \cdot \xi_{2}\right)\left(u+\frac{1}{4}\right)+\frac{1}{2}\left(\xi_{1} \cdot \xi_{2}\right)\left(u+\frac{1}{4}\right)\left(s+\frac{1}{4}\right)\right]
\end{aligned}
$$


The extra factor of $i$ in $\mathcal{A}_{2}$ is coming from the extra factor of $x_{45}=2 i y$ in this amplitude. The functions $L_{1}, L_{2}, L_{3}$ are the following :

$$
\begin{aligned}
& L_{1}=(2)^{-2(t+s+u)-1} \pi \frac{\Gamma\left(-u+\frac{3}{4}\right) \Gamma\left(-s+\frac{3}{4}\right) \Gamma(-t) \Gamma(-t-s-u)}{\Gamma\left(-u-t+\frac{3}{4}\right) \Gamma\left(-t-s+\frac{3}{4}\right) \Gamma\left(-s-u+\frac{1}{2}\right)} \\
& L_{2}=(2)^{-2(t+s+u)-1} \pi \frac{\Gamma\left(-u+\frac{3}{4}\right) \Gamma\left(-s-\frac{1}{4}\right) \Gamma(-t+1) \Gamma(-t-s-u)}{\Gamma\left(-u-t+\frac{3}{4}\right) \Gamma\left(-t-s+\frac{3}{4}\right) \Gamma\left(-s-u+\frac{1}{2}\right)} \\
& L_{3}=(2)^{-2(t+s+u)} \pi \frac{\Gamma\left(-u+\frac{1}{4}\right) \Gamma\left(-s+\frac{1}{4}\right) \Gamma\left(-t+\frac{1}{2}\right) \Gamma\left(-t-s-u-\frac{1}{2}\right)}{\Gamma\left(-u-t+\frac{3}{4}\right) \Gamma\left(-t-s+\frac{3}{4}\right) \Gamma\left(-s-u+\frac{1}{2}\right)}
\end{aligned}
$$

From the poles of the gamma functions, one realizes that the scattering amplitude has tachyon, massless and infinite number of massive poles. To compare the field theory which has tachyon and massless fields e.g., the WZ action, with the above amplitude, one must expand the amplitude such that the tachyon and massless poles of the field theory survive and all other poles disappear in the form of contact terms. In the next section we will find such expansion.

\section{Momentum expansion}

Using the momentum conservation along the world volume of brane, $k_{1}^{i}+k_{2}^{i}+k_{3}^{i}+p^{i}=0$, one finds the Mandelstam variables satisfy

$$
s+t+u=-p_{i} p^{i}-1 / 4
$$

In general, it has been argued in [18] that the momentum expansion of a S-matrix element should be around $\left(k_{i}+k_{j}\right)^{2} \rightarrow 0$ and/or $k_{i} \cdot k_{j} \rightarrow 0$. The case $\left(k_{i}+k_{j}\right)^{2} \rightarrow 0$ is when there is massless pole in $\left(k_{i}+k_{j}\right)^{2}$-channel. One can easily observe that the amplitude (5) must

have massless pole only in $\left(k_{1}+k_{2}\right)^{2}$-channel, so the momentum expansion must be around

$$
k_{3} . k_{1} \rightarrow 0, \quad k_{3} \cdot k_{2} \rightarrow 0, \quad\left(k_{1}+k_{2}\right)^{2} \rightarrow 0
$$

Using the on-shell relations $k_{1}^{2}=k_{2}^{2}=0$ and $k_{3}^{2}=1 / 4$, one can rewrite it in terms of the Mandelstam variables as

$$
s \rightarrow-1 / 4, \quad u \rightarrow-1 / 4, \quad t \rightarrow 0
$$

The constraint (17) then indicates that $p_{i} p^{i} \rightarrow 1 / 4$ which is possible only for euclidean brane. This is consistent with the observation made in [19, 4] that the on-shell condition implies that the S-matrix element can be evaluated only for non-BPS SD-branes [20]. 
Expansion of the functions $L_{1}, L_{2}, L_{3}$ around the above point is

$$
\begin{aligned}
L_{1}= & -\pi^{3 / 2}\left(\frac{1}{t} \sum_{n=-1}^{\infty} b_{n}(u+s+1 / 2)^{n+1}\right. \\
& \left.+\sum_{p, n, m=0}^{\infty} e_{p, n, m} t^{p}((s+1 / 4)(u+1 / 4))^{n}(s+u+1 / 2)^{m}\right) \\
L_{2}= & -\pi^{3 / 2}\left(\frac{1}{(s+1 / 4)} \sum_{n=-1}^{\infty} b_{n}(u+t+1 / 4)^{n+1}\right. \\
& \left.+\sum_{p, n, m=0}^{\infty} e_{p, n, m}(s+1 / 4)^{p}(t(u+1 / 4))^{n}(t+u+1 / 4)^{m}\right) \\
L_{3}= & -\pi^{5 / 2}\left(\sum_{n=0}^{\infty} c_{n}(s+t+u+1 / 2)^{n}\right. \\
& +\frac{\sum_{n, m=0}^{\infty} c_{n, m}\left[(s+1 / 4)^{n}(u+1 / 4)^{m}+(s+1 / 4)^{m}(u+1 / 4)^{n}\right]}{(t+s+u+1 / 2)} \\
& \left.+\sum_{p, n, m=0}^{\infty} f_{p, n, m}(s+t+u+1 / 2)^{p}\left[(s+u+1 / 2)^{n}((s+1 / 4)(u+1 / 4))^{m}\right]\right)
\end{aligned}
$$

where the coefficients $b_{n}$ are exactly the coefficients that appear in the momentum expansion of the S-matrix element of one RR, one gauge field and one tachyon vertex operators [4]. Some of the coefficients $b_{n}, e_{p, n, m}, c_{n}, c_{n, m}$ and $f_{p, n, m}$ are

$$
\begin{aligned}
& b_{-1}=1, b_{0}=0, b_{1}=\frac{1}{6} \pi^{2}, b_{2}=2 \zeta(3) \\
& e_{2,0,0}=e_{0,1,0}=2 \zeta(3), e_{1,0,0}=\frac{1}{6} \pi^{2}, e_{1,0,2}=\frac{19}{60} \pi^{4}, e_{1,0,1}=e_{0,0,2}=6 \zeta(3) \\
& e_{0,0,1}=\frac{1}{3} \pi^{2}, e_{3,0,0}=\frac{19}{360} \pi^{4}, e_{0,0,3}=e_{2,0,1}=\frac{19}{90} \pi^{4}, e_{1,1,0}=e_{0,1,1}=\frac{1}{30} \pi^{4} \\
& c_{0}=0, c_{1}=\frac{\pi^{2}}{3}, c_{2}=4 \xi(3), c_{1,1}=\frac{\pi^{2}}{3}, c_{0,0}=1 \\
& c_{1,0}=c_{0,1}=0, c_{3,0}=c_{0,3}=0 c_{2,0}=c_{0,2}=\frac{\pi^{2}}{3}, c_{1,2}=c_{2,1}=-8 \xi(3) \\
& f_{0,1,0}=-\frac{2 \pi^{2}}{3}, f_{0,2,0}=-f_{1,1,0}=12 \xi(3), f_{0,0,1}=4 \xi(3)
\end{aligned}
$$

$L_{1}$ has massless pole in $t$-channel, $L_{2}$ has tachyonic pole in $s$-channel and $L_{3}$ has tachyonic pole in $(s+t+u)$-channel. These poles must be reproduced in field theory by appropriate couplings. The string amplitude (16) is non-zero for $p=n+3, p=n-1$ and for $p=n+1$. Let us study each case separately. 


\section{$3.1 p=n+3$ case}

This is the simplest case to consider. Only $\mathcal{A}_{1}$ in (16) is non-zero. The trace in $\mathcal{A}_{1}$ is:

$$
\operatorname{Tr}\left(H_{(n)} M_{p} \Gamma^{j l i m}\right)= \pm \frac{32}{n !} \epsilon^{j l i m i_{0} \cdots i_{p-4}} H_{i_{0} \cdots i_{p-4}}
$$

We are going to compare string theory S-matrix elements with field theory S-matrix elements including their coefficients, however, we are not interested in fixing the overall sign of the amplitudes. Hence, in above and in the rest of equations in this paper, we have payed no attention to the overall sign of equations. The string amplitude for electric RR field then becomes

$$
\mathcal{A}^{A A T C}=\mp \frac{32 i}{(p-3) !}\left(\mu_{p}^{\prime} \beta^{\prime} \pi^{1 / 2}\right) \operatorname{Tr}\left(\lambda_{1} \lambda_{2} \lambda_{3}\right) \xi_{1 i} \xi_{2 j} k_{1 m} k_{2 l} \epsilon^{j l i m i_{0} \cdots i_{p-4}} H_{i_{0} \cdots i_{p-4}}(t+s+u+1 / 2) L_{3}
$$

where we have also normalized the amplitude by $\left(\mu_{p}^{\prime} \beta^{\prime} \pi^{1 / 2}\right)$. Apart from the group factor the above amplitude is antisymmetric under interchanging the gauge fields. So the whole amplitude is zero for abelian gauge group. The amplitude also satisfies the Ward identity, i.e., the amplitude vanishes under replacing each of $\xi^{i} \rightarrow k^{i}$. Since $(t+s+u+1 / 2) L_{3}$ has no tachyon/massless pole, the amplitude has only contact terms. The leading contact term is reproduced by the following coupling:

$$
\beta^{\prime} \mu_{p}^{\prime}\left(2 \pi \alpha^{\prime}\right)^{3} \operatorname{Tr}\left(C_{p-4} \wedge F \wedge F \wedge D T\right)
$$

and the non-leading order terms should be corresponding to the higher derivative extension

of the above coupling. This coupling is exactly given by the WZ terms (2) after expanding the exponential and using the multiplication rule of the supermatrices [4].

\section{$3.2 p=n-1$ case}

The next simple case to consider is $p=n-1$. Only $\mathcal{A}_{3}$ in (16) is non-zero for this case. The trace in this amplitude is:

$$
\operatorname{Tr}\left(H_{(n)} M_{p}\right)= \pm \frac{32}{n !} \epsilon^{i_{0} \cdots i_{p}} H_{i_{0} \cdots i_{p}}
$$

Substituting this trace in $\mathcal{A}_{3}$, one finds

$$
\begin{aligned}
\mathcal{A}^{A A T C}= & \mp \frac{32 i}{(p+1) !}\left(\beta^{\prime} \mu_{p}^{\prime} \pi^{1 / 2}\right) \operatorname{Tr}\left(\lambda_{1} \lambda_{2} \lambda_{3}\right) \epsilon^{i_{0} \cdots i_{p}} H_{i_{0} \cdots i_{p}} L_{3}\left\{-t\left(k_{3} \cdot \xi_{1}\right)\left(k_{3} \cdot \xi_{2}\right)\right. \\
& \left.+\left(k_{3} \cdot \xi_{2}\right)\left(k_{2} \cdot \xi_{1}\right)\left(s+\frac{1}{4}\right)+\left(k_{3} \cdot \xi_{1}\right)\left(k_{1} \cdot \xi_{2}\right)\left(u+\frac{1}{4}\right)+\frac{1}{2}\left(\xi_{1} \cdot \xi_{2}\right)\left(u+\frac{1}{4}\right)\left(s+\frac{1}{4}\right)\right\}
\end{aligned}
$$


where we have also normalized the amplitude by $\left(\beta^{\prime} \mu_{p}^{\prime} \pi^{1 / 2}\right)$. The amplitude satisfies the Ward identity and it is symmetric under interchanging the gauge fields. So the amplitude is non-zero even for abelian case.

All terms in (22) have tachyon pole in the $(s+t+u)$-channel and infinite contact terms. We consider only the tachyon pole and show that they can be reproduced by WZ coupling $C_{p} \wedge D T$ and the higher derivative two-gauge-two-tachyon couplings that have been found in [21]. To this end, consider the amplitude for decaying one R-R field to two gauge fields and one tachyon in the world-volume theory of the non-BPS branes which is given by the following Feynman amplitude :

$$
\mathcal{A}=V^{\alpha}\left(C_{p}, T\right) G^{\alpha \beta}(T) V^{\beta}\left(T, T_{3}, A_{1}, A_{2}\right)
$$

where the tachyon propagator and the vertex $V^{\alpha}\left(C_{p}, T\right)$ are given as

$$
\begin{aligned}
G^{\alpha \beta}(T) & =\frac{i \delta^{\alpha \beta}}{\left(2 \pi \alpha^{\prime}\right) T_{p}\left(-k^{2}-m^{2}\right)} \\
V^{\alpha}\left(C_{p}, T\right) & =2 i \mu_{p}^{\prime} \beta^{\prime}\left(2 \pi \alpha^{\prime}\right) \frac{1}{(p+1) !} \epsilon^{i_{0} \cdots i_{p}} H_{i_{0} \cdots i_{p}} \operatorname{Tr}\left(\Lambda^{\alpha}\right)
\end{aligned}
$$

In above vertex, $\operatorname{Tr}\left(\Lambda^{\alpha}\right)$ is non-zero only for abelian matrix $\Lambda^{\alpha}$. The vertex $V^{\beta}\left(T, T_{3}, A_{1}, A_{2}\right)$ can be derived from the higher derivative of two-gauge-two-tachyon couplings [21] (equation (29) of [21]). They are the higher derivative extension of two-gauge-two-tachyon couplings of the tachyon action (3). Using the fact that the off-shell tachyon is abelian, one finds the vertex $V^{\beta}\left(T, T_{3}, A_{1}, A_{2}\right)$ to be

$$
\begin{aligned}
& 2 i T_{p}\left(\pi \alpha^{\prime}\right)\left(\alpha^{\prime}\right)^{2+n+m}\left(a_{n, m}+b_{n, m}\right) \operatorname{Tr}\left(\lambda_{1} \lambda_{2} \lambda_{3} \Lambda^{\beta}\right)\left[-t\left(k_{3} \cdot \xi_{1}\right)\left(k_{3} \cdot \xi_{2}\right)+\left(k_{3} \cdot \xi_{2}\right)\left(k_{2} \cdot \xi_{1}\right)\left(s+\frac{1}{4}\right)\right. \\
& \left.+\left(k_{3} \cdot \xi_{1}\right)\left(k_{1} \cdot \xi_{2}\right)\left(u+\frac{1}{4}\right)+\frac{1}{2}\left(\xi_{1} \cdot \xi_{2}\right)\left(u+\frac{1}{4}\right)\left(s+\frac{1}{4}\right)\right]\left(\left(k_{3} \cdot k_{1}\right)^{n}\left(k_{3} \cdot k_{2}\right)^{m}+\left(k_{3} \cdot k_{1}\right)^{n}\left(k_{1} \cdot k\right)^{m}\right. \\
& +\left(k \cdot k_{2}\right)^{m}\left(k \cdot k_{1}\right)^{n}+\left(k_{1} \cdot k\right)^{n}\left(k_{3} \cdot k_{1}\right)^{m}+\left(k_{3} \cdot k_{2}\right)^{m}\left(k_{2} \cdot k\right)^{n}+\left(k \cdot k_{2}\right)^{n}\left(k_{1} \cdot k\right)^{m}+\left(k_{3} \cdot k_{2}\right)^{n} \\
& \left.\times\left(k_{1} \cdot k_{3}\right)^{m}+\left(k_{3} \cdot k_{2}\right)^{n}\left(k_{2} \cdot k\right)^{m}\right)
\end{aligned}
$$

where $k$ is the momentum of the off-shell tachyon. There are similar terms which have coefficient $\operatorname{Tr}\left(\lambda_{2} \lambda_{1} \lambda_{3} \Lambda^{\beta}\right)$. Some of the coefficients $a_{n, m}$ and $b_{n, m}$ are [21]

$$
\begin{gathered}
a_{0,0}=-\frac{\pi^{2}}{6}, b_{0,0}=-\frac{\pi^{2}}{12} \\
a_{1,0}=2 \zeta(3), a_{0,1}=0, b_{0,1}=b_{1,0}=-\zeta(3) \\
a_{1,1}=a_{0,2}=-7 \pi^{4} / 90, a_{2,0}=-4 \pi^{4} / 90, b_{1,1}=-\pi^{4} / 180, b_{0,2}=b_{2,0}=-\pi^{4} / 45 \\
a_{1,2}=a_{2,1}=8 \zeta(5)+4 \pi^{2} \zeta(3) / 3, a_{0,3}=0, a_{3,0}=8 \zeta(5), \\
b_{0,3}=-4 \zeta(5), b_{1,2}=-8 \zeta(5)+2 \pi^{2} \zeta(3) / 3
\end{gathered}
$$


and $b_{n, m}$ is symmetric.

Now one can write $k_{1} \cdot k=k_{2} \cdot k_{3}-\left(k^{2}+m^{2}\right) / 2$ and $k_{2} \cdot k=k_{1} \cdot k_{3}-\left(k^{2}+m^{2}\right) / 2$. The terms $k^{2}+m^{2}$ in the vertex (25) will be canceled with the $k^{2}+m^{2}$ in the denominator of the tachyon propagator resulting a bunch of contact terms of one RR, two gauge fields and one tachyon in which we are not interested. Ignoring them, one finds the following tachyon pole :

$$
\begin{aligned}
& -32 \pi \alpha^{\prime 2} \beta^{\prime} \mu_{p}^{\prime} \frac{\epsilon^{i_{0} \cdots i_{p}} H_{i_{0} \cdots i_{p}}}{(p+1) !\left(s^{\prime}+t+u^{\prime}\right)} \operatorname{Tr}\left(\lambda_{1} \lambda_{2} \lambda_{3}\right) \sum_{n, m=0}^{\infty}\left(\left(a_{n, m}+b_{n, m}\right)\left[s^{\prime m} u^{\prime n}+s^{\prime n} u^{\prime m}\right]\right. \\
& \left.\times\left[-t\left(k_{3} \cdot \xi_{2}\right)\left(k_{3} \cdot \xi_{1}\right)+\left(k_{2} \cdot \xi_{1}\right)\left(k_{3} \cdot \xi_{2}\right) s^{\prime}+\left(k_{1} \cdot \xi_{2}\right)\left(k_{3} \cdot \xi_{1}\right) u^{\prime}+\left(\xi_{1} \cdot \xi_{2}\right) \frac{1}{2} u^{\prime} s^{\prime}\right]\right)
\end{aligned}
$$

where $u^{\prime}=u+1 / 4=-\alpha^{\prime} k_{2} \cdot k_{3}$ and $s^{\prime}=s+1 / 4=-\alpha^{\prime} k_{1} \cdot k_{3}$. The above amplitude should be compared with the tachyon pole in (22). Let us compare them for some values of $n, m$. For $n=m=0$, the amplitude (27) has the following numerical factor:

$$
-8\left(a_{0,0}+b_{0,0}\right)=-8\left(\frac{-\pi^{2}}{6}+\frac{-\pi^{2}}{12}\right)=2 \pi^{2}
$$

Similar term in (22) has the numerical factor $\left(2 \pi^{2} c_{0,0}\right)$ which is equal to the above number. At the order of $\alpha^{\prime}$, the amplitude (27) has the following numerical factor:

$$
-4\left(a_{1,0}+a_{0,1}+b_{1,0}+b_{0,1}\right)\left(s^{\prime}+u^{\prime}\right)=0
$$

Similar term in (22) is proportional to $\pi^{2} c_{1,0}(s+u+1 / 2)$ which is zero. At the order of $\left(\alpha^{\prime}\right)^{2}$, the amplitude (27) has the following factor :

$$
\begin{aligned}
& -8\left(a_{1,1}+b_{1,1}\right)\left(s^{\prime}\right)\left(u^{\prime}\right)-4\left(a_{0,2}+a_{2,0}+b_{0,2}+b_{2,0}\right)\left[\left(s^{\prime}\right)^{2}+\left(u^{\prime}\right)^{2}\right] \\
& =\frac{\pi^{4}}{3}\left(2 s^{\prime} u^{\prime}\right)+\frac{2 \pi^{4}}{3}\left(s^{\prime 2}+u^{\prime 2}\right)
\end{aligned}
$$

Similar term in (22) has numerical factor $\pi^{2} c_{1,1}\left(2 s^{\prime} u^{\prime}\right)+\pi^{2}\left(c_{2,0}+c_{0,2}\right)\left(s^{\prime 2}+u^{\prime 2}\right)$ which is equal to the above factor using the coefficients (20). At the order of $\alpha^{\prime 3}$, this amplitude has the following factor :

$$
\begin{aligned}
& -4\left(a_{3,0}+a_{0,3}+b_{0,3}+b_{3,0}\right)\left[\left(s^{\prime}\right)^{3}+\left(u^{\prime}\right)^{3}\right]-4\left(a_{1,2}+a_{2,1}+b_{1,2}+b_{2,1}\right)\left[\left(s^{\prime}\right)\left(u^{\prime}\right)\left(s^{\prime}+u^{\prime}\right)\right] \\
& =-16 \pi^{2} \xi(3) s^{\prime} u^{\prime}\left(s^{\prime}+u^{\prime}\right)
\end{aligned}
$$

which is equal to corresponding term in (22) , i.e., $\pi^{2}\left(c_{0,3}+c_{3,0}\right)\left[\left(s^{\prime}\right)^{3}+\left(u^{\prime}\right)^{3}\right]+\pi^{2}\left(c_{2,1}+\right.$ $\left.c_{1,2}\right) s^{\prime} u^{\prime}\left(s^{\prime}+u^{\prime}\right)$. Similar comparison can be done for all order of $\alpha^{\prime}$. Hence, the field theory amplitude (27) reproduces exactly the infinite tower of the tachyon pole of string theory amplitude (22). This indicates that the momentum expansion of the amplitude $C A A T$ is consistent with the momentum expansion of the amplitude TTAA found in [21]. 


\section{$3.3 p=n+1$ case}

We finally consider the case $p=n+1$. Only $\mathcal{A}_{2}$ in (16) is non-zero for this case. The trace in this amplitude is:

$$
\operatorname{Tr}\left(H_{(n)} M_{p} \Gamma^{i j}\right)= \pm \frac{32}{n !} \epsilon^{i_{0} \cdots i_{p-2} i j} H_{i_{0} \cdots i_{p-2}}
$$

Substituting this trace in $\mathcal{A}_{2}$, one finds

$$
\begin{aligned}
\mathcal{A}^{A A T C}= & \mp \frac{32}{(p-1) !}\left(\mu_{p}^{\prime} \beta^{\prime} \pi^{1 / 2}\right) \operatorname{Tr}\left(\lambda_{1} \lambda_{2} \lambda_{3}\right) H_{i_{0} \cdots i_{p-2}} \epsilon^{i_{0} \cdots i_{p}}\left\{\left(2 k_{2} \cdot \xi_{1} k_{2 i_{p-1}} \xi_{2 i_{p}}\right.\right. \\
& -2 k_{1} \cdot \xi_{2} k_{1 i_{p-1}} \xi_{1 i_{p}}+2 k_{1} \cdot \xi_{2} \xi_{1 i_{p-1}} k_{2 i_{p}}+2 k_{2} \cdot \xi_{1} \xi_{2 i_{p}} k_{1 i_{p-1}}-t \xi_{1 i_{p}} \xi_{2 i_{p-1}} \\
& \left.\left.+2 \xi_{1} \cdot \xi_{2} k_{1 i_{p}} k_{2 i_{p-1}}\right) L_{1}+\left(-2 k_{3} \cdot \xi_{1} k_{2 i_{p-1}} \xi_{2 i_{p}} L_{2}-1 \leftrightarrow 2\right)\right\}
\end{aligned}
$$

where again we have normalized the amplitude by $\left(\mu_{p}^{\prime} \beta^{\prime} \pi^{1 / 2}\right)$. Apart from the group factor the amplitude is antisymmetric under interchanging the gauge fields, so the whole amplitude is zero for abelian gauge group. The amplitude satisfies the Ward identity. The first six terms have contact terms as well as massless pole in t-channel and the last two terms in (28) have contact terms as well as tachyon poles in s-channel and u-channel. We are going to analyze all order of the tachyon/massless poles and the leading order and next to the leading order contact terms in this section. Let us study each case separately.

\subsubsection{Tachyon pole}

We first consider the tachyon pole. Replacing (19) in above amplitude, one finds the following tachyon poles:

$$
\begin{aligned}
\mathcal{A}^{A A T C}= & \mp \frac{32}{(p-1) !}\left(2 \pi^{3 / 2}\right)\left(\mu_{p}^{\prime} \beta^{\prime} \pi^{1 / 2}\right) \operatorname{Tr}\left(\lambda_{1} \lambda_{2} \lambda_{3}\right) H_{i_{0} \cdots i_{p-2}} \epsilon^{i_{0} \cdots i_{p}} \\
& \times \sum_{n=-1}^{\infty} b_{n}\left(\frac{(u+t+1 / 4)^{n+1}}{s+1 / 4}\left(k_{3} \cdot \xi_{1}\right) k_{2 i_{p-1}} \xi_{2 i_{p}}-\frac{(s+t+1 / 4)^{n+1}}{u+1 / 4}\left(k_{3} \cdot \xi_{2}\right) k_{1 i_{p-1}} \xi_{1 i_{p}}\right)
\end{aligned}
$$

and some contact terms that we consider them in section 3.3.3. Since (29) is antisymmetric under interchanging $1 \leftrightarrow 2$, we consider only the first term. This term should be reproduced in field theory by the following Feynman amplitude:

$$
\mathcal{A}=V^{\alpha}\left(C_{p-2}, A_{2}, T\right) G^{\alpha \beta}(T) V^{\beta}\left(T, T_{3}, A_{1}\right)
$$


where the vertices can be found from the standard nonabelian kinetic term of the tachyon and from the higher derivative extension of the WZ coupling $C_{p-2} \wedge F \wedge D T$ found in [4] (equation (16) of [4]), i.e.,

$$
\begin{aligned}
V^{\beta}\left(T, T_{3}, A_{1}\right) & =i T_{p}\left(2 \pi \alpha^{\prime}\right)\left(k_{3}-k\right) \cdot \xi_{1} \operatorname{Tr}\left(\lambda_{3} \lambda_{1} \Lambda^{\beta}\right) \\
V^{\alpha}\left(C_{p-2}, A_{2}, T\right) & =2 \mu_{p}^{\prime} \beta^{\prime} \frac{\left(2 \pi \alpha^{\prime}\right)^{2}}{(p-1) !} \epsilon^{i_{0} \cdots i_{p}} H_{i_{0} \cdots i_{p-2}} k_{2 i_{p-1}} \xi_{2 i_{p}} \sum_{n=-1}^{\infty} b_{n}\left(\alpha^{\prime} k_{2} \cdot k\right)^{n+1} \operatorname{Tr}\left(\lambda_{2} \Lambda^{\alpha}\right)
\end{aligned}
$$

where $k$ is the momentum of the off-shell tachyon. Note that the vertex $V^{\beta}\left(T, T_{3}, A_{1}\right)$ has no higher derivative correction as it arises from the kinetic term of the tachyon. The amplitude (30) then becomes

$$
\begin{aligned}
\mathcal{A}= & 4 \mu_{p}^{\prime} \beta^{\prime}\left(2 \pi \alpha^{\prime}\right)^{2} \frac{1}{(p-1) !\left(s+\frac{1}{4}\right)} \operatorname{Tr}\left(\lambda_{1} \lambda_{2} \lambda_{3}\right) \epsilon^{i_{0} \cdots i_{p}} H_{i_{0} \cdots i_{p-2}} k_{2 i_{p-1}} \xi_{2 i_{p}}\left(k_{3} \cdot \xi_{1}\right) \\
& \times \sum_{n=-1}^{\infty} b_{n}\left(\frac{\alpha^{\prime}}{2}\right)^{n+1}(t+u+1 / 4)^{n+1}
\end{aligned}
$$

which is exactly the tachyon pole of the string theory amplitude (29).

\subsubsection{Massless pole}

We now consider the massless pole. Replacing the expansion of $L_{1}$ into (28), one finds the following massless pole in $t$-channel :

$$
\begin{aligned}
\mathcal{A}^{A A T C}= & \pm \frac{32 \mu_{p}^{\prime} \beta^{\prime} \pi^{2}}{t(p-1) !} \operatorname{Tr}\left(\lambda_{1} \lambda_{2} \lambda_{3}\right) H_{i_{0} \cdots i_{p-2}} \epsilon^{i_{0} \cdots i_{p}} \sum_{n=-1}^{\infty} b_{n}(u+s+1 / 2)^{n+1}\left[2 k_{2} \cdot \xi_{1} k_{2 i_{p-1}} \xi_{2 i_{p}}\right. \\
& \left.-2 k_{1} \cdot \xi_{2} k_{1 i_{p-1}} \xi_{1 i_{p}}+2 k_{1} \cdot \xi_{2} \xi_{1 i_{p-1}} k_{2 i_{p}}+2 k_{2} \cdot \xi_{1} \xi_{2 i_{p}} k_{1 i_{p-1}}+2 \xi_{1} \cdot \xi_{2} k_{1 i_{p}} k_{2 i_{p-1}}\right]
\end{aligned}
$$

and some contact terms that we consider them in section 3.3.3. In field theory, the massless pole is given by the following Feynman amplitude :

$$
\mathcal{A}=V_{\alpha}^{i}\left(C_{p-2}, T_{3}, A\right) G_{\alpha \beta}^{i j}(A) V_{\beta}^{j}\left(A, A_{1}, A_{2}\right)
$$

The vertices and propagator are

$$
\begin{aligned}
V_{\alpha}^{i}\left(C_{p-2}, T_{3}, A\right) & =2 \mu_{p}^{\prime} \beta^{\prime}\left(2 \pi \alpha^{\prime}\right)^{2} \frac{1}{(p-1) !} \epsilon^{i_{0} \cdots i_{p-1} i} H_{i_{0} \cdots i_{p-2}} k_{i_{p-1}} \sum_{n=-1}^{\infty} b_{n}\left(\alpha^{\prime} k_{3} \cdot k\right)^{n+1} \operatorname{Tr}\left(\lambda_{3} \Lambda^{\alpha}\right) \\
V_{\beta}^{j}\left(A, A_{1}, A_{2}\right) & =-i T_{p}\left(2 \pi \alpha^{\prime}\right)^{2} \operatorname{Tr}\left(\lambda_{1} \lambda_{2} \Lambda_{\beta}\right)\left[\xi_{1}^{j}\left(k_{1}-k\right) \cdot \xi_{2}+\xi_{2}^{j}\left(k-k_{2}\right) \cdot \xi_{1}+\xi_{1} \cdot \xi_{2}\left(k_{2}-k_{1}\right)^{j}\right] \\
G_{\alpha \beta}^{i j}(A) & =\frac{i \delta_{\alpha \beta} \delta^{i j}}{\left(2 \pi \alpha^{\prime}\right)^{2} T_{p}(t)}
\end{aligned}
$$


where $k$ is momentum of the off-shell gauge field. Here again the vertex $V_{\alpha}^{i}\left(C_{p-2}, T_{3}, A\right)$ has been found from the higher derivative extension of the WZ coupling $C_{p-2} \wedge F \wedge D T$ that has been found in [4]. Note again that the vertex $V_{\beta}^{j}\left(A, A_{1}, A_{2}\right)$ has no higher derivative correction as it arises from the kinetic term of the gauge field. Replacing them in the amplitude (32), one finds

$$
\begin{aligned}
\mathcal{A}= & \left(2 \pi \alpha^{\prime}\right)^{2} \frac{2 \mu_{p}^{\prime} \beta^{\prime}}{(p-1) ! t} \epsilon^{i_{0} \cdots i_{p-1} i} H_{i_{0} \cdots i_{p-2}} \operatorname{Tr}\left(\lambda_{1} \lambda_{2} \lambda_{3}\right) \sum_{n=-1}^{\infty} b_{n}\left(\frac{\alpha^{\prime}}{2}\right)^{n+1}(s+u+1 / 2)^{n+1} \\
& \times\left(2\left(k_{2} \cdot \xi_{1}\right) k_{1 i_{p-1}} \xi_{2 i}-2\left(k_{1} \cdot \xi_{2}\right) k_{1 i_{p-1}} \xi_{1 i}-2\left(k_{1} \cdot \xi_{2}\right) k_{2 i_{p-1}} \xi_{1 i}+2\left(k_{2} \cdot \xi_{1}\right) \xi_{2 i} k_{2 i_{p-1}}\right. \\
& \left.-2\left(\xi_{1} \cdot \xi_{2}\right) k_{1 i_{p-1}} k_{2 i}\right)
\end{aligned}
$$

where we have used $\sum_{\alpha} \lambda_{i j}^{\alpha} \lambda_{k l}^{\alpha}=\delta_{i k} \delta_{j l}$. This is exactly the massless pole of the string theory amplitude (31). This indicates that the momentum expansion of the S-matrix element $C A A T$ in this paper is consistent with the momentum expansion of the S-matrix element $C A T$ found in [4].

\subsubsection{Contact terms}

Replacing (19) into (28), one finds the following contact terms at leading order and next to the leading order:

$$
\begin{aligned}
\mathcal{A}^{A A T C}= & \mp \frac{32}{(p-1) !}\left(\mu_{p}^{\prime} \beta^{\prime} \pi^{2}\right) \operatorname{Tr}\left(\lambda_{1} \lambda_{2} \lambda_{3}\right) H_{i_{0} \cdots i_{p-2}} \epsilon^{i_{0} \cdots i_{p}}\left\{\xi_{1 i_{p}} \xi_{2 i_{p-1}}-\frac{\pi^{2}}{6}\left(2 k_{2} \cdot \xi_{1} k_{2 i_{p-1}} \xi_{2 i_{p}}\right.\right. \\
& -2 k_{1} \cdot \xi_{2} k_{1 i_{p-1}} \xi_{1 i_{p}}+2 k_{1} \cdot \xi_{2} \xi_{1 i_{p-1}} k_{2 i_{p}}+2 k_{2} \cdot \xi_{1} \xi_{2 i_{p}} k_{1 i_{p-1}}-t \xi_{1 i_{p}} \xi_{2 i_{p-1}} \\
& \left.+2 \xi_{1} \cdot \xi_{2} k_{1 i_{p}} k_{2 i_{p-1}}\right)[t+2(s+u+1 / 2)]+\frac{\pi^{2}}{6} \xi_{1 i_{p}} \xi_{2 i_{p-1}}(s+u+1 / 2)^{2} \\
& \left.+\left(\frac{\pi^{2}}{3} k_{3} \cdot \xi_{1} k_{2 i_{p-1}} \xi_{2 i_{p}}[2(t+u+1 / 4)+s+1 / 4]-[1 \leftrightarrow 2]\right)\right\}
\end{aligned}
$$

The first term is reproduced by $C A A T$ coupling of the following gauge invariant coupling:

$$
2 \beta^{\prime} \mu_{p}^{\prime}\left(2 \pi \alpha^{\prime}\right)^{2} \operatorname{Tr}\left(C_{p-2} \wedge F \wedge D T\right)
$$

which is exactly given by the WZ terms (2) after expanding the exponential and using the multiplication rule of the supermatrices [4]. The other terms in (34) should be related to the higher derivative extension of the above coupling. However, there are many other higher derivative gauge invariant couplings which have contribution to the contact terms of the S-matrix element of $C A A T$. Comparing them with the string theory contact terms (34), 
one can not fix their coefficients uniquely. One particular set of higher derivative gauge invariant couplings that reproduce the contact terms in (34) are the following:

$$
\begin{gathered}
-\frac{1}{12} \beta^{\prime} \mu_{p}^{\prime}\left(2 \pi \alpha^{\prime}\right)^{4}\left[-i D^{\beta} F_{a \alpha} D^{\alpha} F_{b \beta} D_{c} T+\frac{3 i}{2} F_{a c} D_{\alpha} F_{\beta b} D^{\alpha} D^{\beta} T-\frac{3 i}{2} D_{\alpha} F_{\beta b} F_{a c} D^{\alpha} D^{\beta} T\right. \\
-\frac{1}{2} D_{a} D^{\alpha} D_{c} F_{b \alpha} D_{\beta} D^{\beta} T+F_{a \alpha} D^{\beta} D^{\alpha} D_{\beta} D_{b} D_{c} T-\frac{1}{2} D_{a} D^{\alpha} D_{\beta} D^{\beta} F_{b \alpha} D_{c} T \\
+D_{b} D_{c} F_{a \alpha} D^{\beta} D^{\alpha} D_{\beta} T+4 D^{\alpha} D_{a} D_{c} F_{\beta b} D_{\alpha} D^{\beta} T-\frac{1}{2} D_{a} F_{\alpha \beta} D_{b} D^{\alpha} D^{\beta} D_{c} T \\
-D_{a} D^{\beta} D_{\beta} D_{c} F_{b \alpha} D^{\alpha} T+2 D_{b} D^{\alpha} D^{\beta} F_{a \alpha} D_{\beta} D_{c} T+D^{\alpha} D_{\alpha} D_{c} F_{\beta b} D^{\beta} D_{a} T \\
+D_{a} D^{\beta} D_{\beta} F_{b \alpha} D^{\alpha} D_{c} T+\frac{1}{2} D^{\beta} D^{\alpha} D_{\beta} D_{c} F_{a \alpha} D_{b} T \\
\left.-\frac{1}{2} D^{\alpha} D^{\beta} F_{a b} D_{\alpha} D_{\beta} D_{c} T\right] \frac{1}{(p-2) !} C_{i_{0} \cdots i_{p-3}} \epsilon^{i_{0} \cdots i_{p-3} a b c}
\end{gathered}
$$

where $D_{a} T=\partial_{a} T-i\left[A_{a}, T\right]$. Among the couplings in (36), only the last coupling has nonzero on-shell $C T A$ coupling. This coupling has been found in [4] from the S-matrix element of one RR, one gauge and one tachyon vertex operators. This coupling has been also used in the previous section to verify that the tachyon/massless poles in (28) are reproduced by the higher derivative couplings in field theory. All coupling in (36) are at $\left(\alpha^{\prime}\right)^{4}$ order. The next order terms should be at $\left(\alpha^{\prime}\right)^{5}$ order, and so on.

As we have mentioned in the Introduction section, the WZ couplings can also be found using the BSFT. In that framework, it has been argued in [2] when the RR field is constant, there is no higher derivative correction to the WZ couplings. So one may expect that the above higher derivative WZ couplings should be zero for constant RR field. However, as we have mentioned before, the above couplings are valid when $p_{i} p^{i} \rightarrow 1 / 4$. So they can not be compared with the $p_{i} p^{i}=0$ result of the BSFT.

\section{Acknowledgment}

E.H would like to thank A.Ghodsi for useful comments.

\section{References}

[1] A. Sen, Int. J. Mod. Phys. A 20, 5513 (2005) arXiv:hep-th/0410103.

[2] P. Kraus and F. Larsen, Phys. Rev. D 63, 106004 (2001) arXiv:hep-th/0012198.

[3] T. Takayanagi, S. Terashima and T. Uesugi, JHEP 0103, 019 (2001) arXiv:hep-th/0012210. 
[4] M. R. Garousi, arXiv:0802.2784 [hep-th].

[5] M. R. Garousi and E. Hatefi, Nucl. Phys. B 800, 502 (2008) arXiv:0710.5875 [hepth]].

[6] M. R. Garousi, JHEP 0812, 059 (2008) [arXiv:0810.2256 [hep-th]].

[7] M. R. Garousi, arXiv:0811.4334 [hep-th].

[8] A. Sen, JHEP 9910, 008 (1999) arXiv:hep-th/9909062.

[9] M. R. Garousi, Nucl. Phys. B 584, 284 (2000) arXiv:hep-th/0003122].

[10] E. A. Bergshoeff, M. de Roo, T. C. de Wit, E. Eyras and S. Panda, JHEP 0005, 009 (2000) arXiv:hep-th/0003221.

[11] J. Kluson, Phys. Rev. D 62, 126003 (2000) arXiv:hep-th/0004106.

[12] C. Kennedy and A. Wilkins, Phys. Lett. B 464, 206 (1999) arXiv:hep-th/9905195|.

[13] A. Sen, arXiv:hep-th/9904207.

[14] P. J. De Smet and J. Raeymaekers, JHEP 0008, 020 (2000) arXiv:hep-th/0004112.

[15] M. R. Garousi and R. C. Myers, Nucl. Phys. B 475, 193 (1996) arXiv:hep-th/9603194.

[16] H. Liu and J. Michelson, Nucl. Phys. B 614, 330 (2001) arXiv:hep-th/0107172.

[17] A. Fotopoulos, JHEP 0109, 005 (2001) arXiv:hep-th/0104146].

[18] M. R. Garousi, JHEP0802, 169 (2008) arXiv:0712.1954 [hep-th].

[19] M. Billo, B. Craps and F. Roose, JHEP 9906, 033 (1999) arXiv:hep-th/9905157.

[20] M. Gutperle and A. Strominger, JHEP 0204, 018 (2002) arXiv:hep-th/0202210];

[21] M. R. Garousi and H. Golchin, Nucl. Phys. B 800, 547 (2008) arXiv:0801.3358 [hep-th]. 